\title{
VECTOR FIELDS ON SOME CLASS OF COMPLETE SYMMETRIC VARIETIES
}

\author{
YOSHIFUMI KATO
}

\section{§1. Introduction}

In the previous papers [6], [7], we show that the set of an algebraic homogeneous space $G / P$ fixed under the action of a maximal torus $T$ can be canonically identified with the coset $W^{1}=W / W_{1}$ of Weyl group $W$. We find a $T$ invariant Zariski open set near each element $w \in W^{1}$ and introduce a very nice local coordinate system such that we can express the maximal torus action explicitly. As a result, we become able to apply the study of J. B. Carrell and D. Lieberman [2], [3] to the space $G / P$ and investigate the numerical properties of its characteristic classes and cycles.

The main purpose of this paper is to show that some class of complete symmetric varieties, see the definition in C. DeConcini and C. Procesi [4], [5], have also a nice local coordinate system as above. The canonical compactification of a complex semisimple Lie group of adjoint type belongs to this class. Therefore our results may be related to some combinatorial problems.

\section{§2. Complete symmetric varieties}

To fix the terminology which will be needed later, we recall some results about complete symmetric varieties. See [4], [5].

Let $G$ be a simply connected complex semisimple Lie group with an automorphism $\sigma$ of order two. Let $H$ be the group consisting of all $\sigma$ fixed elements. We denote by $\mathfrak{g}$ and $\mathfrak{h}$ the Lie algebras which correspond to $G$ and $H$ respectively. Let $\tilde{H}$ be the normalizer of $H$ in $G$ then it is of finite index $[\tilde{H} ; H]=2^{s}$ for some integer $s$. For any subgroup $H^{\prime}$ satisfying $H \subset H^{\prime} \subset \tilde{H}$, C. DeConcini and C. Procesi call the homogeneous space $G / H^{\prime}$ a symmetric variety and seek the canonical compactification of $G / \tilde{H}$.

Received January 8, 1985. 
Let $T$ be a $\sigma$ fixed maximal torus whose Lie algebra is $t$. The automorphism $\sigma$ acts canonically on $t$ and decompose it as $t=t_{0} \oplus t_{1}$ where $t_{0}$ (resp. $t_{1}$ ) corresponds to the eigenvalues +1 (resp. -1 ). The Lie algebra $t_{0}$ is that of the torus $T_{0}=T^{\sigma}$ while $t_{1}$ is that of the torus $T_{1}=\left\{t \in T \mid t^{\sigma}\right.$ $\left.=t^{-1}\right\}$. The product mapping $T_{0} \times T_{1} \rightarrow T$ gives an isogeny. Hereafter we assume that we choose the torus $T$ for which $\operatorname{dim} T_{1}$ is maximal among all possible $\sigma$ stable tori. We put $\ell$ to be the dimension of $T_{1}$ and call it the rank of the symmetric variety $G / H$.

Let $\Phi \subset t^{*}$ be the root system and

$$
\mathfrak{g}=\mathfrak{t} \oplus \sum_{\alpha \in \Phi} \mathfrak{g}_{\alpha}
$$

be the root space decomposition. Since $\sigma\left(\mathfrak{g}_{\alpha}\right)=\mathfrak{g}_{\alpha \sigma}$ holds, $\Phi$ is $\sigma$ invariant. Moreover it is known that we can decompose $\Phi$ into positive roots and negative ones as $\Phi=\Phi^{+} \cup \Phi^{-}$satisfying the following condition;

If $\alpha \in \Phi^{+}$and $\alpha \neq 0$ on $t_{1}$ then $\alpha^{\sigma} \in \Phi^{-}$.

We assume this condition and put

$$
\begin{aligned}
& \Phi_{0}=\left\{\alpha \in \Phi \mid \alpha \equiv 0 \text { on } t_{1}\right\}, \\
& \Phi_{1}=\Phi-\Phi_{0} .
\end{aligned}
$$

Then we have

$$
\mathfrak{h}=\mathfrak{t}_{0} \oplus \sum_{\alpha \in \Phi_{0}} \mathfrak{g}_{\alpha} \oplus \sum_{\alpha \in \Phi_{1}} C\left(X_{\alpha}+\sigma\left(X_{\alpha}\right)\right)
$$

where $X_{\alpha}$ is a nonzero element of $\mathfrak{g}_{\alpha}, \alpha \in \Phi_{1}$. Since the subspace

$$
\mathfrak{t}_{1} \oplus \sum_{\alpha \in \Phi_{1-}^{-}} C X_{\alpha}
$$

is a complement of $\mathfrak{h}$ in $g$, the dimension $m$ of the space $G / H$ is equal to $\operatorname{dim} t_{1}+\frac{1}{2}\left|\Phi_{1}\right|=\ell+\frac{1}{2}\left|\Phi_{1}\right|$. Let $\Gamma \subset \Phi^{+}$be the set of simple roots and put

$$
\begin{aligned}
& \Gamma_{0}=\Gamma \cap \Phi_{0}=\left\{\beta_{1}, \cdots, \beta_{k}\right\}, \\
& \Gamma_{1}=\Gamma \cap \Phi_{1}=\left\{\alpha_{1}, \cdots, \alpha_{j}\right\} .
\end{aligned}
$$

Here we order $\alpha_{1}, \cdots, \alpha_{j}$ so that $\alpha_{i}-\alpha_{i}^{\sigma}$ are mutually distinct for $i \leq \ell$ and for each $i>\ell$ there is an index $s \leq \ell$ such that $\alpha_{i}-\alpha_{i}^{\alpha}=\alpha_{s}-\alpha_{s}^{\alpha}$. We put $\bar{\alpha}_{i}=\frac{1}{2}\left(\alpha_{i}-\alpha_{i}^{\sigma}\right)$ for $1 \leq i \leq \ell$ and call them the restricted simple roots.

C. DeConcini and C. Procesi show that the symmetric variety $G / \tilde{H}$ admits a canonical compactification $\underline{\bar{X}}$, called a complete symmetric variety, by using the representation theory of $G$ as follows. Let $\lambda$ be a regular 
special weight and $V_{2 \lambda}$ the space of irreducible representation of $G$ with the highest weight $2 \lambda$. Then $V_{2 \lambda}$ contains a unique non zero element $h^{\prime}$ fixed under $H$ up to non zero factor. Let $P_{2 \lambda}=P\left(V_{2 \lambda}\right)$ be the projective space of lines in $V_{2 \lambda}$ and $\tilde{h} \in P_{2 \lambda}$ be the class of $h^{\prime}$. Since the $G$ orbit $G \tilde{h}$ of $\tilde{h}$ is isomorphic to the space $G / \tilde{H}$, if we take its closure $\bar{X}=\overline{G \tilde{h}}$ in $P_{2 \lambda}$, we can obtain a $G$ equivariant compactification of $G / \tilde{H}$. And we can show that $\underline{\bar{X}}$ is independent of the choice of $\lambda$ and has nice properties. The following is fundamental.

1) $\underline{\bar{X}}$ is smooth.

2) $\underline{\bar{X}}-G \tilde{h}$ is a union of $\ell$ smooth hypersurfaces $S_{i}, 1 \leq i \leq \ell$, which cross transversely.

3) The $G$ orbits of $\underline{\bar{X}}$ correspond to the subsets of indices $1,2, \cdots, \ell$ so that the orbit closures are the intersections $S_{i_{1}} \cap \cdots \cap S_{i_{k}}$.

4) The unique closed orbit $Y \simeq G / P$ is $S_{1} \cap \cdots \cap S_{\ell}$ where $P$ is the parabolic subgroup of $G$ which keeps the class $\left[v_{2 \lambda}\right]$ representing the highest weight vector $v_{2 \lambda}$ invariant.

The inclusion $i: Y=G / P G \bar{X}$ induces the homomorphism $i^{*}: \operatorname{Pic}(\underline{\bar{X}}) \rightarrow$ $\operatorname{Pic}(Y)$ and then $i^{*}$ is injective. Therefore if the induced line bundle of $L \in \operatorname{Pic}(\underline{\bar{X}})$ corresponds to the weight $\lambda$, we can denote $L$ by $L_{\lambda}$. The hypersurfaces $S_{i}, 1 \leq i \leq \ell$, are given by the equation $y_{i}=0$, where $y_{i}$ is the $i$-th coordinate function on $V$ in the next section, and the line bundles $O\left(S_{i}\right)$ are isomorphic to $L_{2 \bar{x} i}$.

The closure of the $T_{1}$ orbit $T_{1} \tilde{h}$ is isomorphic to $\ell$ dimensional affine space $C^{l}$. The isomorphism is given by the mappings;

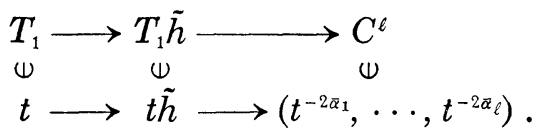

And $T_{1} \tilde{h}$ can be identified with the open set $\left(C^{*}\right)^{\ell}$ in $C^{\ell}$. We decompose $V_{2 \lambda}=C v_{2 \lambda} \oplus \tilde{V}_{2 \lambda}$ in a $T$ stable way and put $A=v_{2 \lambda} \oplus \tilde{V}_{2 \lambda}, V=A \cap \underline{\bar{X}}$. The set $V$ contains $\tilde{h}$ and so $\overline{T_{1} \tilde{h}} \simeq C^{\ell}$. The origin of $C^{\ell}$ corresponds to the highest weight vector $v_{2 \lambda}$. Let $U$ be the unipotent subgroup corresponding to $\Phi_{1}^{-}$. Then the mapping

$$
\begin{array}{r}
U \times \overline{T_{1} \tilde{h}} \underset{\cup}{\simeq} U \times C^{\ell} \longrightarrow \underset{\cup}{V} \\
(u, x) \longrightarrow u \cdot x
\end{array}
$$

gives an isomorphism. In the next section, we will show that the Zariski open set $V$ in $\underline{X}$ has excellent properties. 


\section{§3. Vector fields on $\underline{\bar{X}}$}

We choose $\ell$ elements $A_{1}, \cdots, A_{\ell}$ of $t_{1}$ such that $\bar{\alpha}_{i}\left(A_{j}\right)=-\frac{1}{2} \delta_{i, j}, 1 \leq i$, $j \leq \ell$ and identify the $T_{1}$ orbit $T_{1} \tilde{h}$ and $\left(C^{*}\right)^{\ell}$ by the following mappings;

$$
\begin{gathered}
T_{1} \longrightarrow T_{1} \tilde{h} \longrightarrow \underset{\Psi}{\Psi} \\
t_{1}=\exp \left(\sum_{i=1}^{\ell} a_{i} A_{i}\right) \longrightarrow \exp \left(\sum_{i=1}^{\ell} a_{i} A_{i}\right) \tilde{h} \longrightarrow \underset{\left(y_{i=1}^{\ell}\right.}{\longrightarrow} \longrightarrow C^{\ell}
\end{gathered}
$$

where

$$
y_{i}=\exp a_{i}, \quad 1 \leq i \leq \ell .
$$

Since $t_{1}^{-2 a_{j}}=\exp \left(-2 \sum_{i=1}^{\ell} a_{i} \bar{\alpha}_{j}\left(A_{i}\right)\right)=\exp a_{i}$, this identification is the same with that of (2.6). The functions $\left(y_{i}\right)_{i=1}^{\ell}$ can be extended as the canonical coordinate of the affine space $C^{\ell}$.

We can decompose an element $J \in \mathfrak{t}$ uniquely as $J=J_{0}+J_{1}, J_{0} \in t_{0}$, $J_{1} \in t_{1}$, and then $J_{0}=\frac{1}{2}\left(J+J^{\sigma}\right), J_{1}=\frac{1}{2}\left(J-J^{\sigma}\right)$.

LEMma 1. We have

$$
J_{1}=-2 \sum_{i=1}^{\ell} \bar{\alpha}_{i}(J) A_{i}
$$

Proof. We put

$$
J_{1}=\frac{1}{2}\left(J-J^{\sigma}\right)=\sum_{i=1}^{\ell} a_{i} A_{i}
$$

Then

$$
\begin{aligned}
\bar{\alpha}_{j}\left(J_{1}\right) & =\frac{1}{4}\left(\alpha_{j}-\alpha_{j}^{\sigma}\right)\left(J-J^{\sigma}\right) \\
& =\frac{1}{2}\left(\alpha_{j}-\alpha_{j}^{\sigma}\right)(J)=\bar{\alpha}_{j}(J) .
\end{aligned}
$$

On the other hand

$$
\bar{\alpha}_{j}\left(J_{1}\right)=\sum_{i=1}^{\ell} a_{i} \bar{\alpha}_{j}\left(A_{i}\right)=-\frac{1}{2} a_{j}
$$

hence we have

$$
a_{j}=-2 \bar{\alpha}_{j}(J)
$$

Let $\mathfrak{u}$ be the Lie algebra of the group $U$. We choose a non zero element $X_{r} \in \mathfrak{g}_{r}$ for $\gamma \in \Phi_{1}^{-}$and take $\left\{X_{r}\right\}_{r \in \Phi_{1}^{-}}$as a basis of $\mathfrak{u}$. We write as $Z=$ $\sum_{r \in \Phi_{1}^{-}} z_{\gamma} X_{r} \in \mathfrak{H}$. Let $N(T)$ be the normalizer of $T$ in $G$. For any element $w$ of $N(T)$, we can consider the following diagram; 


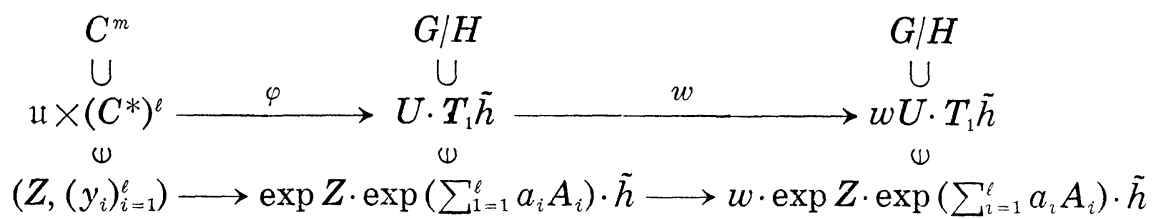

where

$$
y_{i}=\exp a_{i}, \quad 1 \leq i \leq \ell .
$$

Since $\mathfrak{u}$ is nilpotent, the mapping $Z \rightarrow \exp Z \in U$ is one-to-one and onto. From (2.7), the mapping $\varphi$ is not only isomorphic but also it can be extended to the isomorphism $\tilde{\varphi}$ as follows

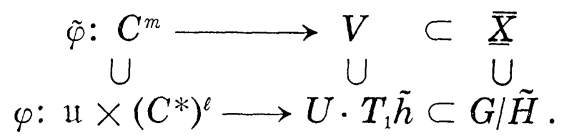

Since the left multiplication of $w$ is obviously one-to-one, the pair ( $w V$, $\tilde{\varphi}^{-1} \circ w^{-1}$ ) gives a local coordinate on the Zariski open set $w V$. Since the origin of $C^{m}$ corresponds to the class $\left[v_{2 \lambda}\right] \in P_{2 \lambda}$ of the highest weight vector $v_{2 \lambda}$, we can consider that $w$ represents the class $\left[v_{2 w \alpha}\right] \in P_{2 \lambda}$ of the extremal weight vector $v_{2 w \lambda}$. Since $w \in N(T) \cap P$ keeps the set $V$ invariant, it is sufficient to run $w$ over the representatives of $W^{1}=N(T) / N(T) \cap P$ in $N(T)$. For brevity we use the same letter $w$ for the coset of $W^{1}$ which $w$ represents.

TheOREM 2. Let $\underline{\bar{X}}$ be the complete symmetric variety that is the canonical compactification of a symmetric variety $G / \tilde{H}$. Let $w V, w \in W^{1}$, be the Zariski open set defined as above. Then $w V$ is invariant under the left action of the maximal torus $T$. Indeed if we use the local coordinate ( $w V$, $\left.\tilde{\varphi}^{-1} \circ w^{-1}\right)$ and act an element $\exp J \in T, J \in \mathrm{t}$, the coordinate changes from

$$
\left\{\left(\boldsymbol{z}_{\gamma}\right)_{\gamma \in \Phi_{1}^{-}},\left(y_{i}\right)_{i=1}^{\ell}\right\}
$$

to

$$
\left\{\left(\exp (w \gamma)(J) z_{\gamma}\right)_{r \in \Phi_{\overline{1}}^{-}},\left(\exp \left(-2\left(w \bar{\alpha}_{i}\right)(J)\right) y_{i}\right)_{i=1}^{\ell}\right\} .
$$

Remark. If $\underline{\bar{X}}$ is covered completely with $w V, w \in W^{1}$, we show that $\underline{X}$ admits a very nice local coordinate system as an algebraic homogeneous space $G / P$. See [6], [7]. We give a necessary and sufficient condition in the next proposition.

Proof. We take $\exp J \in T, J \in$ t. Then we have 


$$
\begin{aligned}
& \exp J \cdot w \exp Z \cdot \exp \left(\sum_{i=1}^{\ell} a_{i} A_{i}\right) \tilde{h} \\
& \quad=w \cdot w^{-1} \exp J w \cdot \exp Z \cdot w^{-1} \exp (-J) w \cdot w^{-1} \exp J w \cdot \exp \left(\sum_{i=1}^{\ell} a_{i} A_{i}\right) \tilde{h} \\
& \quad=w \cdot \exp w^{-1}(J) \cdot \exp Z \cdot \exp \left(-w^{-1}(J)\right) \cdot \exp w^{-1}(J) \cdot \exp \left(\sum_{i=1}^{\ell} a_{i} A_{i}\right) \tilde{h} \\
& =w \cdot \exp \left(\operatorname{Ad}\left(\exp w^{-1}(J)\right) Z\right) \cdot \exp \left(-2 \sum_{i=1}^{\ell} \bar{\alpha}_{i}\left(w^{-1}(J)\right)\right) \cdot t^{\prime} \cdot \exp \left(\sum_{i=1}^{\ell} a_{i} A_{i}\right) \tilde{h} \\
& \quad=w \cdot \exp \left(\operatorname{Exp}\left(\operatorname{ad} w^{-1}(J)\right) Z\right) \cdot \exp \left(\sum_{i=1}^{\ell}\left(-2\left(w \bar{\alpha}_{i}\right)(J)+a_{i}\right) A_{i}\right) \tilde{h}
\end{aligned}
$$

Here we put

$$
y_{i}=\exp a_{i}, \quad 1 \leq i \leq \ell,
$$

and use the decomposition

$$
\exp \left(w^{-1}(J)\right)=\exp \left(-2 \sum_{i=1}^{\ell} \bar{\alpha}_{i}\left(w^{-1}(J)\right)\right) \cdot t^{\prime}
$$

where

$$
\exp \left(-2 \sum_{i=1}^{\ell} \bar{\alpha}_{i}\left(w^{-1}(J)\right)\right) \in T_{1}, \quad t^{\prime} \in T_{0}
$$

Since

$$
\begin{aligned}
\operatorname{ad}\left(w^{-1}(J)\right) Z & =\left[w^{-1}(J), \sum_{r \in \Phi_{\overline{-}}^{-}} z_{r} X_{r}\right] \\
& =\sum_{r \in \Phi_{\overline{1}}^{-}}(w \gamma)(J) z_{r} X_{r}
\end{aligned}
$$

we have

$$
\operatorname{Exp}\left(\operatorname{ad}\left(w^{-1}(J)\right)\right) Z=\sum_{r \in \Phi_{\overline{1}}^{-}} \exp ((w \gamma)(J)) z_{\gamma} X_{r} \in \mathfrak{U} .
$$

This completes the proof.

We define a vector field $V_{J}$ on $X$ by the following rule

$$
\left(V_{J} f\right)(p)=\lim _{\varepsilon \rightarrow 0} \frac{1}{\varepsilon}(f(\exp (\varepsilon J) p)-f(p))
$$

where $J \in t, p \in \underline{\bar{X}}$ and $f$ is a function near $p$. Then from the above theorem, if we use the local coordinate $\left(w V, \tilde{\varphi}^{-1} \circ w^{-1}\right), V_{J}$ can be written explicitly on $w V$ as follows

$$
V_{J}=\sum_{\gamma \in \Phi_{\bar{T}}^{-}}(w \gamma)(J) z_{\gamma} \frac{\partial}{\partial z_{\gamma}}-2 \sum_{i=1}^{\ell}\left(w \bar{\alpha}_{i}\right)(J) y_{i} \frac{\partial}{\partial y_{i}} .
$$


Therefore if we choose $J$ such that

$$
\begin{array}{ll}
(w \gamma)(J) \neq 0, & \gamma \in \Phi_{1}^{-}, \\
\left(w \bar{\alpha}_{i}\right)(J) \neq 0, & 1 \leqq i \leqq \ell,
\end{array}
$$

$V_{J}$ vanishes at the point $w \in w V$ in the first order.

Proposition 3. The complete symmetric variety $\bar{X}$ is covered with the Zariski open sets $w V, w \in W^{1}$ i.e., $\bar{X}=\bigcup_{w \in W^{1}} w V$, if and only if all the $T$ fixed points of $\bar{X}$ are contained in the minimal $G$ orbit $Y \simeq G / P \subseteq \underline{\bar{X}}$.

Proof. Since there is the unique $T$ fixed point $w$ in the set $w V$ and $w$ belongs to $Y$, if $\underline{\bar{X}}=\bigcup_{w \in W^{1}} w \mathrm{~V}$ holds, all the $T$ fixed points are contained in $Y$. We can show the contrary by following the same discussion used in [6]. If all the $T$ fixed points of $\underline{\bar{X}}$ are contained in the minimal $G$ orbit $Y \simeq G / P$, they are identified with the set $W^{1}$. And if $\bar{X}^{\prime}=\underline{X}-$ $\bigcup_{w \in W^{1}} w V$ is not empty, since $\bar{X}^{\prime}$ is a $T$ invariant closed variety, it must have a $T$ fixed point. See [6] or [9]. But this point can not belong to $W^{1}$. This is a contradiction.

Example 4. Let $G^{\prime}$ be a simply connected complex semisimple Lie group and put $G=G^{\prime} \times G^{\prime}$. The automorphism $\sigma$ in $G$ of order two is given by the exchange

$$
\sigma(x, y)=(y, x) \quad x, y \in G^{\prime},
$$

and then

$$
H=\left\{(x, x) \mid x \in G^{\prime}\right\} .
$$

The space $G / \tilde{H}$ can be identified with the group $G^{\prime \prime}=G^{\prime} / C\left(G^{\prime}\right)$ where $C\left(G^{\prime}\right)$ is the center of $G^{\prime}$. In other words, $G^{\prime \prime}$ is a complex semisimple Lie group of adjoint type. In this case $\underline{\bar{X}}$ is covered with the Zariski open sets $w V$, $w \in W^{1}$. The set $W^{1}$ is the direct product $W^{1}=W^{\prime} \times W^{\prime}$ of the Weyl group $W^{\prime}$ of $G^{\prime}$. See [4].

Hereafter we assume the following;

$$
\text { (Ass.) } \quad \underline{\bar{X}}=\bigcup_{w \in W 1} w V \text {. }
$$

But it is an interesting problem to seek a $T$ invariant Zariski open set near each $T$ fixed point concretely when (Ass.) fails. The existence of such an open set comes from the theory of torus embeddings, that is, Sumihiro's theorem. 
We assign one more condition to the choice of $J$

(II) $(w r)(J), r \in \Phi_{1}^{-}$, and $\left(w \bar{\alpha}_{i}\right)(J), 1 \leq i \leq \ell$, are all real .

TheOREM 5. If $\underline{\bar{X}}$ satisfies (Ass.) then the dimension $h^{p, q}$ of $H^{q}\left(X, \Omega^{p}\right)$ are determined as follows;

1) $h^{p, q}=0$ for $p \neq q$,

2) $h^{p, p}=\left\{\begin{array}{l}w \in W^{1} \\ \begin{array}{l}\text { values among }(w \gamma)(J), \gamma \in \Phi_{1}^{-}, \\ -\left(w \bar{\alpha}_{i}\right)(J), 1 \leq i \leq \ell, \text { are positive }\end{array}\end{array}\right\}$.

Proof. Since $\underline{\bar{X}}$ is a compact kähler manifold with a holomorphic vector field whose zero set are isolated but not empty, 1) follows. See [3]. Since the eigenvalues of the Lie derivative $L_{V_{J}}$ at $w \in W^{1}$ are $\{2(w r)(J)$, $\left.\gamma \in \Phi_{1}^{-},-4\left(w \bar{\alpha}_{i}\right)(J), 1 \leq i \leq \ell\right\}$, if we use the result of [8], which is used in [6], we obtain 2).

\section{§4. Relations to the paper [3]}

Let $M$ be a $n$ dimensional compact kähler manifold with a holomorphic vector field $V$ whose zero set $Z=\operatorname{Zero}(V)$ is isolated but not empty. Then J. B. Carrell and D. Lieberman show in [3] that there exists a filtration in $H^{\circ}\left(Z, \mathcal{O}_{Z}\right)$ where the sheaf $\mathcal{O}_{Z}$ is $\mathcal{O}_{X} / i(V) \Omega^{1}$ such that

$$
\begin{aligned}
& \text { 1) } H^{\circ}\left(Z, \mathcal{O}_{Z}\right)=F_{-n} \supset F_{-n+1} \supset \cdots \supset F_{-1} \supset F_{0} \supset\{0\}, \\
& \text { 2) } F_{i} \cdot F_{j} \subset F_{i+j}, \\
& \text { 3) } F_{-k} / F_{-k+1} \simeq H^{k}\left(M, \Omega^{k}\right), \\
& \text { 4) } \operatorname{gr} H^{0}\left(Z, \mathcal{O}_{Z}\right)=\bigoplus_{k=0}^{n} F_{-k} / F_{-k+1} \simeq H^{*}(M, C) .
\end{aligned}
$$

If the zero set $Z$ is simple then we can write $Z=\left\{\zeta_{1}, \cdots, \zeta_{m}\right\}$ and consider that $H^{\circ}\left(Z, \mathcal{O}_{Z}\right)$ is the vector space $C \zeta_{1} \oplus \cdots \oplus C \zeta_{m}$ of complex valued functions on $Z$.

Let $E$ be a vector bundle on $M$ of rank $r$. The bundle $E$ is, by definition, $V$ equivariant if it admits a $C$-linear homomorphism $\tilde{V}: E \rightarrow E$ satisfying

$$
\tilde{V}(f \cdot s)=(V f) \cdot s+f \cdot \tilde{V} s
$$

where $s$ is a local section of $E$ and $f$ is a function. If we restrict $\tilde{V}$ to $\tilde{V}_{z}=\left.\tilde{V}\right|_{z}$ on $Z, \tilde{V}_{z}$ defines a homomorphism $\tilde{V}_{z} \in \operatorname{Hom}\left(E_{z}, E_{z}\right)$ where $E_{z}$ $=E \otimes \mathcal{O}_{z}$. And the representative of $d$-th Chern classes, $0 \leq d \leq n$, of $E$ in $F_{-d}$ are given by the formula 


$$
(-1)^{d} \sigma_{d}\left(\tilde{V}_{Z}\right) \in H^{0}\left(Z, \mathcal{O}_{Z}\right)
$$

Here $\sigma_{d}$ is the usual $d$-th elementary function defined by

$$
\operatorname{det}|t I+A|=\sum_{d=0}^{r} \sigma_{d}(A) t^{r-a}, \quad A \in \operatorname{Hom}\left(E_{Z}, E_{Z}\right) .
$$

We apply these results to the complete symmetric variety $\underline{\bar{X}}$ satisfying (Ass.).

Theorem 6. Let $T(\underline{\bar{X}})$ be the tangent bundle of $\bar{X}$ then it is $V_{J}$ equivariant. The representative of $d$-th Chern classes, $0 \leq d \leq m$, of $T(\bar{X})$ in $F_{-d}$ is the following

$$
\begin{aligned}
& W^{1}=Z=\operatorname{Zero}\left(V_{J}\right) \longrightarrow C \\
& \Psi \\
& w \longrightarrow(-1)^{d} \sigma_{d}\left((-(w \gamma)(J))_{r \in \Phi_{1}^{-}},\left(2\left(w \bar{\alpha}_{i}\right)(J)\right)_{i=1}^{\ell}\right) .
\end{aligned}
$$

Proof. For a local vector field $v \in T(\underline{\bar{X}})$, we define

$$
\tilde{V}_{J} v=\left[V_{J}, v\right]
$$

then $\tilde{V}_{J}$ satisfies (4.5). Hence it is $V_{J}$ equivariant. If we use the local coordinate $\left(w V, \tilde{\varphi}^{-1} \circ w^{-1}\right), w \in W^{1}$, we have

$$
\begin{aligned}
\tilde{V}_{J} \frac{\partial}{\partial z_{\gamma}} & =\left[\sum_{\xi \in \Phi_{1}^{-}}(w \xi)(J) z_{\xi} \frac{\partial}{\partial z_{\xi}}-2 \sum_{j=1}^{\ell}\left(w \bar{\alpha}_{j}\right)(J) y_{j} \frac{\partial}{\partial y_{j}}, \frac{\partial}{\partial z_{\gamma}}\right] \\
& =-(w \gamma)(J) \frac{\partial}{\partial z_{\gamma}}, \quad \gamma \in \Phi_{1}^{-},
\end{aligned}
$$

and

$$
\begin{aligned}
\tilde{V}_{J} \frac{\partial}{\partial y_{i}} & =\left[\sum_{\xi \in \Phi_{1}^{-}}(w \xi)(J) z_{\xi} \frac{\partial}{\partial z_{\xi}}-2 \sum_{j=1}^{\ell}\left(w \bar{\alpha}_{j}\right)(J) y_{j} \frac{\partial}{\partial y_{j}}, \frac{\partial}{\partial y_{i}}\right] \\
& =2\left(w \bar{\alpha}_{i}\right)(J) \frac{\partial}{\partial y_{i}}, \quad 1 \leq i \leq \ell .
\end{aligned}
$$

Using matrix notation, we complete the proof.

Remark. From this theorem, the representative of Poincaré dual of one point in $F_{-m}$ is given by

$$
\begin{aligned}
& W^{1} \longrightarrow C \\
& \Psi \\
& w \longrightarrow \frac{(-1)^{m}}{\sharp} \sigma_{m}\left((-(w r)(J))_{r \in \Phi_{1}^{-}},\left(2\left(w \bar{\alpha}_{i}\right)(J)\right)_{i=1}^{\ell}\right) .
\end{aligned}
$$

Theorem 7. The line bundles associated with local sections of $O\left(S_{i}\right)$, $1 \leq i \leq \ell$, are $V_{J}$ equivariant. The representatives of the first Chern classes of them in $F_{-1}$ are given by 


$$
\begin{aligned}
& W^{1} \longrightarrow \underset{\psi}{C}, \quad 1 \leq i \leq \ell . \\
& w \quad 2 w \bar{\alpha}_{i}(J)
\end{aligned}
$$

Proof. To make the meanings clear, we denote by $y_{i}^{w}$ the components $y_{i}, 1 \leq i \leq \ell$, of the local coordinate $\left(w V, \tilde{\varphi}^{-1} \circ w^{-1}\right), w \in \Phi_{1}^{-}$. On the Zariski open set $V=e V, y_{i}^{e}=0$ is a local equation of $S_{i}$. Since $S_{i}$ is $G$ stable and hence $W^{1}$ stable, $y_{i}^{w}=0$ gives a local equation of $S_{i}$ on $w V$. For $y_{i}^{w}$, we define

$$
\left(\tilde{V}_{J} y_{i}^{w}\right)(p)=\lim _{\varepsilon \rightarrow 0} \frac{1}{\varepsilon}\left\{y_{i}^{w}(\exp (\varepsilon J) p)-y_{i}^{w}(p)\right\}, \quad p \in w V,
$$

and then

$$
\begin{aligned}
& =\lim _{\varepsilon \rightarrow 0} \frac{1}{\varepsilon}\left\{\exp \left(-2 w \bar{\alpha}_{i}\right)(\varepsilon J) y_{i}^{w}(p)-y_{i}^{w}(p)\right\} \\
& =\left(-2 w \bar{\alpha}_{i}\right)(J) y_{i}^{w}(p) .
\end{aligned}
$$

Therefore $\tilde{V}_{J} y_{i}^{w}$ is also a local section of $S_{i}$ on $w V$. From this property, we can extend it naturally to the $C$-linear homomorphism $\tilde{V}_{J}: O\left(S_{i}\right) \rightarrow$ $O\left(S_{i}\right)$ satisfying (4.5). The first Chern class of it is represented by $w \rightarrow$ $(-1)^{1} \sigma_{1}\left(-2 w \bar{\alpha}_{i}\right)(J)=2 w \bar{\alpha}_{i}(J)$. We complete the proof.

\section{REFERENCES}

[1] Bialynicki-Birula, Some theorems on actions of algebraic groups, Ann. of Math., 98 (1973), 480-497.

[2] J. B. Carrell, Chern classes of the Grassmannians and Schubert calculus, Topology, 17 (1978), 177-182.

[ 3 ] J. B. Carrell and D. Lieberman, Vector fields and Chern numbers, Math. Ann., 225 (1977), 263-273.

[4] C. DeConcini and C. Procesi, Complete symmetric varieties, Springer Lect. Notes, 996, 1-44.

[5] - Complete symmetric varieties II (Intersection theory), Preprint.

[6] Y. Kato, A new characterization of the Bruhat decomposition, Nagoya Math. J., 86 (1982), 131-153.

[ 7 ] - On the vector fields on an algebraic homogeneous space, Pacific J. of Math., 108 (1983), 285-294.

[ 8 ] C. Kosniowsky, Applications of the holomorphic Lefschetz formulae, Bull. London Math. Soc., 2 (1970), 43-48.

[9] A. J. Sommese, Holomorphic vector fields on compact kähler manifolds, Math. Ann., 210 (1974), 75-82.

Department of Mathematical Engineering

Faculty of Engineering

Nagoya University

Chikusa-ku, Nagoya 464

Japan 